\title{
Identification of Poisonous Mushrooms by Means of a Hand-Held Electronic Nose ${ }^{\dagger}$
}

\author{
Francisco Portalo-Calero *, Jesús Lozano *, Félix Meléndez *, Patricia Arroyo * \\ and José Ignacio Suárez * \\ Industrial Engineering School, University of Extremadura, Av. Elvas s/n, 06006 Badajoz, Spain \\ * Correspondence: pacoportalo@unex.es (F.P.-C.); jesuslozano@unex.es (J.L.); felixmv@unex.es (F.M.); \\ parroyoz@unex.es (P.A.); jmarcelo@unex.es (J.I.S.) \\ + Presented at the 8th GOSPEL Workshop. Gas Sensors Based on Semiconducting Metal Oxides: Basic \\ Understanding \& Application Fields, Ferrara, Italy, 20-21 June 2019.
}

Published: 19 June 2019

\begin{abstract}
This work presents a practical application of an electronic nose to fast and efficient discrimination of different species of Amanita mushrooms. The electronic nose instrument were utilized for investigation of discrimination capability with respect to odour profile of these fungi. The home-made prototype was based on MOS-type chemical sensors and headspace sampling method. Samples were cut into thin sheets, placed in glass vials and maintained at a constant temperature using a thermostatic bath, the headspace of which was subjected to analysis. The data were analysed using multivariate methods: PCA, LDA and Artificial Neural Networks. The obtained results confirmed legitimacy of application of the electronic nose technique to identification and discrimination of fungi species. Results show a correct classification of the fungi species at the level of $80-100 \%$.
\end{abstract}

\section{Introduction}

There is no simple test that distinguishes poisonous from nonpoisonous mushrooms, and accurate mushroom identification will require consultation with an experienced mycologist. Although it is estimated that only a few species are lethal, it is not clear how many of the mushrooms worldwide contain potentially toxic compounds. New species are being discovered continuously; for many species, toxicity data are unavailable.

On the other hand, it is known the capacity developed by certain animals to detect volatile substances, and thus find traces of substances emanating from natural products, volatile compounds and biomarkers of different diseases, [1], locate explosives [2] and so on. Among the natural aromas that are able to differentiate, are those released by some types of fungi, traditionally used in their harvest once trained for it. This circumstance suggests the possible existence of different odorous nuances between the different species of mushrooms, beyond those currently recognized by human olfaction.

\section{Material and Methods}

Different mushrooms have been harvested during the autumn months and frozen until the time of analysis. The search location lies between the dehesas of Extremadura (Spain) and the Alentejo (Portugal). The classification was carried out independently by at least two experts. Next, a homemade and home-developed electronic nose (Winose Version 6) was used for the discrimination of the different species. More details of the Winose Version 6 can be found in [3]. 


\section{Results}

Two different tests have performed in this work. In both cases, the first step is to get the mushrooms. Once harvested the samples, they were processed and kept frozen. Later a slice of the same mass is cut from the hat of each mushroom to be used as a sample. Using the headspace technique, at least 15 measurements of each sample were made at a constant temperature $\left(30{ }^{\circ} \mathrm{C}\right)$. The measurement cycle consists of 1 min measuring the sample and a recovery phase of 9 min. This process is repeated for all samples. The measurements were made with a flow rate of 150 (L/min) and the operating temperatures of the sensors have been between 300 and $400{ }^{\circ} \mathrm{C}$.

The main objective of the first test is to check the discrimination capability of the developed electronic nose. In this sense, three different types of Amanita mushrooms were used: phalloides, caesarea, and muscaria. Once measured and processed the data, Principal Component Analysis (PCA) was made for dimensionality reduction in order to plot in Figure 1. A high degree of discrimination for each of them is observed, and it can be assured that the system offers different answers for each one of the samples, being similar for mushrooms of the same species. The results obtained with the PCA were confirmed with the classification by means of Artificial Neural Networks with Backpropagation Learning Algorithm, in which 97.7\% success was obtained in the cross validation using LeaveOneOut method. Only a mistake in the classification of a Muscaria was obtained, since it was classified as Caesarea.

The second test consists of the detection of two species very similar to the sight and that sometimes it is possible to find in nearby locations. These are species of Amanita phalloides and Agaricus silvicola. Once measured and pre-processed data (averaged), Figure 2 graphically represented the response of each sensor, clearly indicated the odorous differences perceived by the enose between both mushrooms.

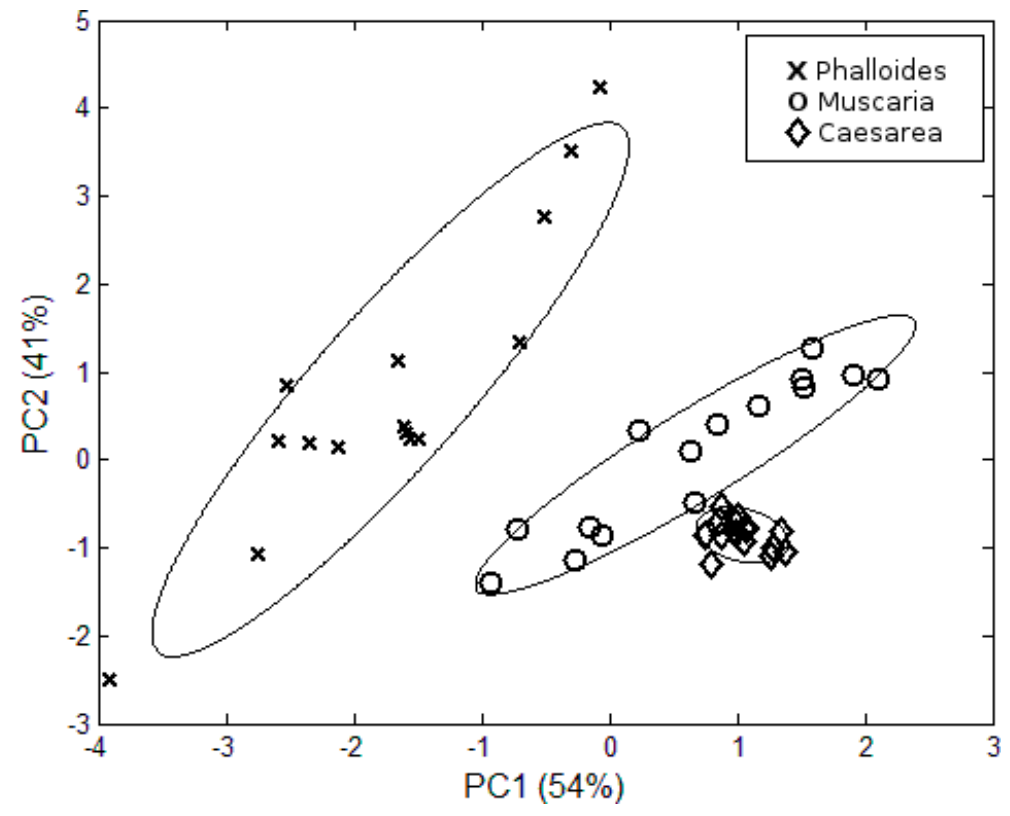

Figure 1. PCA score plot of amanita measurements. 


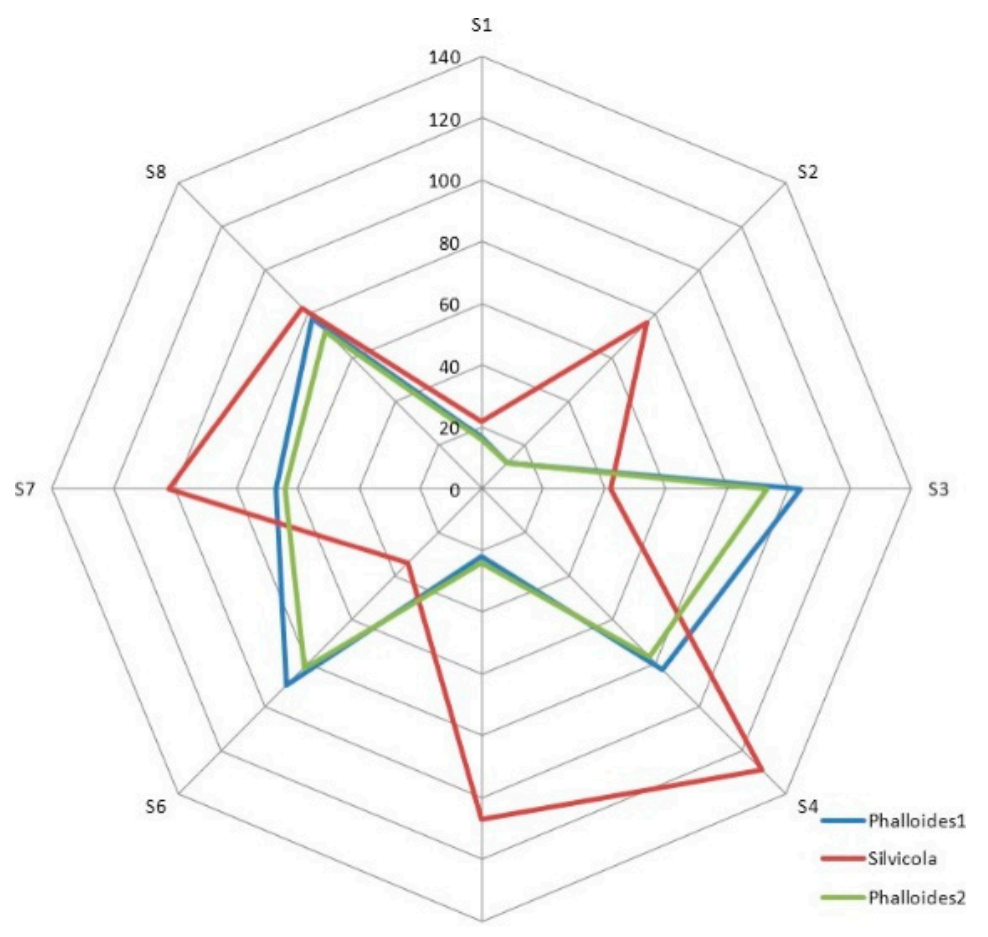

S5

Figure 2. Radial plot of sensors response.

\section{References}

1 Sánchez, C.; Santos, J.P.; Lozano, J. Use of Electronic Noses for Diagnosis of Digestive and Respiratory Diseases through the Breath. Biosensors 2019, 9, 35.

2 Pardo, L.F.M.; Acosta, A.P. Detection of explosives with animal aid: a review of scientific literature. Logos Sci. Technol. Mag. 2009, 1, 106-118.

3 Santos, J.; Aleixandre, M.; Arroyo, P.; Suarez, J.I.; Lozano, J. An Advanced Hand Held Electronic Nose for Ambient Air Applications. Chem. Eng. Trans. 2018, 68, 235-240.

(C) 2019 by the authors. Licensee MDPI, Basel, Switzerland. This article is an open access article distributed under the terms and conditions of the Creative Commons Attribution (CC BY) license (http://creativecommons.org/licenses/by/4.0/). 